\title{
ORIGINAL
}

\section{A novel lipoprotein (a) lowering drug, D-47, decreases neointima thickening after vascular injury}

\author{
Yutaka Nakaya', Daiju Fukuda², Takashi Oyamada'1, Kazuo Ogawa ${ }^{3}$, Nagakatsu Harada', Hironori Nakagami ${ }^{4}$, \\ Ryuichi Morishita ${ }^{4}$, Masataka Sata ${ }^{2}$, and Hiroshi Sakaue ${ }^{1}$
}

${ }^{1}$ Department of Nutrition and Metabolism, and ${ }^{2}$ Department of Cardiology, Institute of Biomedical sciences, Tokushima University Graduate School, Tokushima, Japan, ${ }^{3}$ Pharmaceutical Science, Tokushima Bunri University, Tokushima, Japan and ${ }^{4}$ Department of Clinical Gene Therapy, Graduate School of Medicine, Osaka University, Osaka, Japan

\begin{abstract}
Although $\operatorname{Lp}(a)$ have been thought to be a cardiovascular risk factor, it is unclear whether lowering $\mathrm{Lp}$ (a) levels reduces the risk of cardiovascular diseases. No pharmacological agents which selectively reduce serum $L p(a)$ levels, and $L p(a)$ is present in primate but absent in common laboratory animals such as mice and pigs. In the present study we used transgenic mice of human $L p(a)$ and tested effect a novel $L p(a)$ lowering drug D-47 on neointima formation after vascular injury. D-47 successfully decreased plasma levels of $L p(a)$ and possibly inhibited neointima formation in $\mathrm{Lp}(\mathrm{a})$ transgenic mice. The results indicate that we can modulate plasma Lp(a) levels by pharmacologic agents and inhibit atherogenic properties of $L p(a)$ by reducing plasma levels of Lp(a). J. Med. Invest. 64 : 64-67, February, 2017
\end{abstract}

Keywords : lipoprotein a, atherosclerosis, cardiovascular events, low density lipoprotein

\section{INTRODUCTION}

Lipoprotein(a) $[\mathrm{Lp}(\mathrm{a})]$ is composed of a low density lipoprotein (LDL) particle with apolipoprotein (a) [apo(a)]. Apo(a) is a homolog of plasminogen that contains 10 different types of plasminogen kringle-4-like repeats (kringle- 4 types 1 through 10), suggesting its relation to anti-fibrinolytic properties. Many studies have shown elevated plasma $\mathrm{Lp}$ (a) levels as a risk factor for a variety of atherosclerotic and thrombotic diseases including peripheral vascular disease, thromboembolism, and coronary heart disease $(1,2)$. According to the Adult Treatment Plan (ATP) III guidelines (3), Lp (a) was classified as an "emerging" risk factor for cardiovascular disease. Plasma levels of $\mathrm{Lp}(\mathrm{a})$ are under genetic control and there is no specific agent to reduce plasma Lp(a). Lipid-lowering agents, statins, have little or no effect on plasma $\mathrm{Lp}$ (a) levels. Niacin and estrogen reduce plasma $\mathrm{Lp}$ (a) levels, but only slightly. In addition to lowering $\mathrm{Lp}(\mathrm{a})$, niacin has lowering effects on LDLcholesterol, triglycerides and remnant cholesterol levels. Thus, the benefit of niacin on CVD cannot be mediated only by $\mathrm{Lp}$ (a) reduction. Therefore, highly specific and potent $\mathrm{Lp}(\mathrm{a})$ lowering drugs are awaited to clarify the Lp (a) function.

It is challenging to prove that $\mathrm{Lp}$ (a) lowering can reduce cardiovascular risk in patients with high $\mathrm{Lp}$ (a) levels as well as in animal models. To prove drug effect on $\mathrm{Lp}$ (a) and its function, however, the use of animal models is difficult because of the unusual species distribution of $\operatorname{Lp}(\mathrm{a})$. $\operatorname{Lp}(\mathrm{a})$ is only present in humans and primates, and is absent in common laboratory animals such as mice and pigs (4). In the present study we used double transgenic mice of human $\mathrm{Lp}(\mathrm{a})$, and studied a novel $\mathrm{Lp}$ (a) lowering drug on vascular injury models to study atherogenic action of $\mathrm{Lp}(\mathrm{a})$.

Received for publication October 11, 2016 ; accepted November 9, 2016.

Address correspondence and reprint requests to Yutaka Nakaya, Department of Nutrition and Metabolism, the University of Tokushima, 815 Kuramoto-cho, Tokushima, Japan, 770-8503, and Fax : +81-88-6337113

\section{METHODS}

Drugs

Lp(a) lowering compound, D-47, produced by Dr. K. Ogawa, a co-author of this paper, was used to study Lp(a) function. D-47 is a pharmaceutical formulation composition of solid dispersion contained the arginine salt of S-2E [(S) (+) -4- ((4-tert-Butylphenyl)2-oxo-pyrrolidine-4-yl) methoxybenzoic acid] (5), with "soluplus ${ }^{\mathrm{R}}$ ", (BASF) as the water soluble polymer, in the ratio of one to two. The water solubility and oral absorption of S-2E were improved by this solid dispersion, D-47, which was prepared by solvent method as described below.

The mixture of S-2E arginine salt (30 g) and soluplus (60 g) was dissolved in methanol $250 \mathrm{ml}$ at around $50^{\circ} \mathrm{C}$ and then evaporated the solvent under reduced pressure. Into the residue, ethyl ether $100 \mathrm{ml}$ was added and then evaporated the solvent. The final residue in the vessel was scraped together and then dried under reduced pressure at room temperature to obtain the D-47 (88 g). The amorphous state of S-2E arginine salt in D-47 was confirmed by X-ray powder diffraction analysis and DSC analysis.

\section{Animal}

Eight male $\mathrm{Lp}$ (a) transgenic mice were divided into two groups, control $(n=4)$ and treated groups $(n=4)$. Lp(a) transgenic mice were kindly provided by R. Morishita, Osaka University, Japan (6-8). Because apo(a) is present only in humans, primates and hedgehogs, we used Lp(a) transgenic mice that were generated by crossing human apo (a) transgenic mice (7) and human ApoB transgenic mice (8) to test the drug effects. The mice had free access to water and standard chow (standard non-purified diet, Oriental Yeast, Tokyo, Japan). Mice in the treated group were added D-47(30 mg/kg/day)to the standard chow from 10 weeks (i.e. 2 weeks before surgery) until 4 weeks after surgery. The mice in the control group were treated standard chow which was added only soluplus without D-47. At 12 week, vascular injury was performed to both groups. The experiments were approved by the Ethical Committee for Animal Experiments of the Tokushima University Graduate School of Medicine. 


\section{Production of vascular injury}

Vascular injury was induced in mice as described previously $(9,10)$. A straight spring wire $(0.38 \mathrm{~mm}$ in diameter, No.C-SF-15$15, \mathrm{COOK}$ ) was inserted into the femoral artery and left in place for 1 minute to denude and dilate the artery. Blood flow was restored. Artery was collected at day 28 after the injury. Mice were sacrificed by deep anesthesia and blood was sampled for analysis of lipid profile. For morphometric analysis, the femoral arteries were harvested at 4 weeks after the injury. To investigate the degree of neointimal formation, the proximal segments of the injured arteries were fixed in paraformaldehyde, embedded in paraffin, sectioned at $5 \mu \mathrm{m}$ thickness, and stained with Elastica van Gieson. Neointima and media areas were measured by a computer-assisted image analyzer using Image J Software (BioArts).

\section{Measurement of Lipid profile}

Lp(a) was measured by latex agglutination turbidimetry using a commercial kit (COBAS MIRA, Sanwakagaku Co. Japan). Total cholesterol was measured by Ultra. Violet-End (UV-End) method using cholesterol dehydrogenase, and triglyceride was measured by enzymatic determination using the enzyme glycerol phosphate oxidase (GPO) after hydrolysis by lipoprotein lipase.

\section{Statistics}

The results were expressed as the means \pm standard deviations. The data were compared two groups using Student's t-test. As $\mathrm{Lp}$ (a) levels were variable in each animal, we also used nonparametric comparison (Mann-Whitney U test) between two groups. Values of $\mathrm{P}<0.05$ were considered statistically significant.

\section{RESULTS}

Fig 1 shows the change of the lipid profile and blood glucose levels by the D-47. Treatment of the drug markedly reduced the blood levels of $\mathrm{Lp}$ (a) $(30.3 \pm 12.7 \mathrm{mg} / \mathrm{dL}$ in the control group vs $5.5 \pm 1.3 \mathrm{mg} / \mathrm{dL}$ in the treated group, $\mathrm{p}=0.047 \mathrm{in} \mathrm{U}$-test), and triglyceride $(514.0 \pm 34.2 \mathrm{mg} / \mathrm{dL}$ vs $385.5 \pm 29.3 \mathrm{mg} / \mathrm{dL}, \mathrm{p}=0.003)$. Blood levels of total cholesterol were not altered significantly by D-47 (279.0 $\pm 20.1 \mathrm{mg} / \mathrm{dL}$ vs $284.0 \pm 15.3 \mathrm{mg} / \mathrm{dL}, \mathrm{p}=0.721)$. Blood glucose levels were not different between two groups, either (251.7 $\pm 47.4 \mathrm{mg} / \mathrm{dL}$ vs $327.5 \pm 64.7 \mathrm{mg} / \mathrm{dL}, \mathrm{p}=0.149)$.
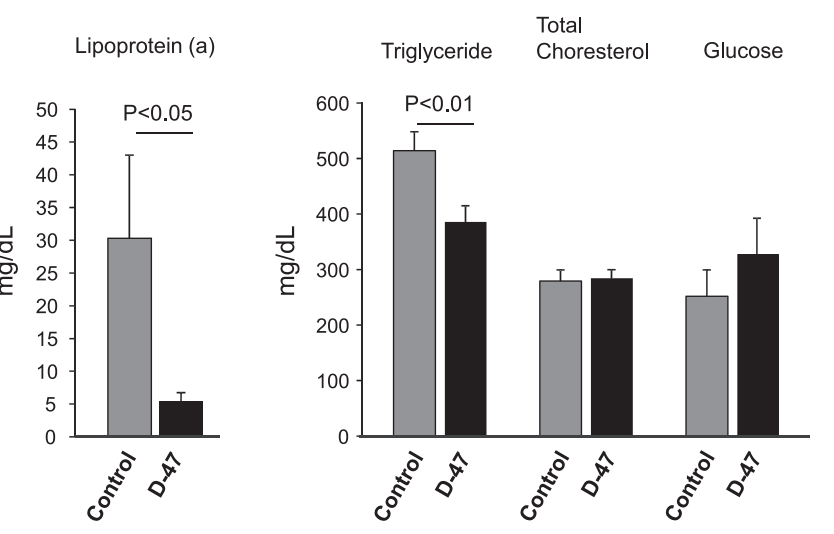

Fig. 1 Effect of D-47 on lipids profile.

Serum levels of lipoprotein (a) were markedly decreased in D-47 treated mice compared to the control mice. Serum total cholesterol and glucose levels were not different between two groups, although serum levels of triglyceride were greater in the D-47 treated mice.
Fig 2 shows the comparison of neointima formation after vascular injury. The neointima areas, which are defined as the area of the space between the lumen and internal elastic lamina, were increased in control mice. In contrast, treatment with D-47 attenuated the neointima areas in transgenic mice (Figure $2 \mathrm{~A}$ and $\mathrm{B}$ ), although the difference did not reach the level of statistical significance $(p=$ 0.175). The ratio of the neointima thickness to the media thickness tended to be smaller in the D- 47 treated mice than in the control mice $(2.12 \pm 0.94$ vs $1.34 \pm 0.36, \mathrm{p}=0.173)$.

\section{DISCUSSION}

$\mathrm{Lp}$ (a) have been thought to be a cardiovascular risk factor. This study demonstrated that a novel $\mathrm{Lp}$ (a) lowering drug, D-47, successfully decreased plasma levels of $\mathrm{Lp}$ (a) and possibly inhibited neointima formation in $\mathrm{Lp}$ (a) transgenic mice. The results indicate that we can modulate plasma Lp(a) levels by pharmacologic agents and inhibit atherogenic properties by reducing plasma levels of $\mathrm{Lp}(\mathrm{a})$.

$\mathrm{Lp}(\mathrm{a})$ is a low density lipoprotein (LDL) particle which is attached to the polypeptide, apo(a). Apo(a) has unique structural properties, i.e., plasminogen kringle-4-like repeats $(1,2)$. Because of the high degree of homology between apo (a) and plasminogen, $\mathrm{Lp}(\mathrm{a}) / \mathrm{apo}$ (a) can competitively inhibit tissue-type plasminogen activator-mediated plasminogen activation on fibrin surfaces, although the mechanism of inhibition by apolipoprotein(a) remains controversial. $\operatorname{Lp}(\mathrm{a})$ and apo (a) also have been thought to enhance the proliferation of human vascular smooth muscle cells (VSMCs) by inhibiting the activation of plasminogen to plasmin $(11,12)$. Further mechanisms have been shown to involve atherogenesity of this lipoprotein.

Lipoprotein apheresis is very efficient in decreasing $\mathrm{Lp}$ (a) concentrations. A single apheresis session can acutely decrease Lp(a) by approximately $60-75 \%$ and weekly or biweekly performed apheresis results in considerably decreased $\mathrm{Lp}$ (a) concentrations. In longitudinal cohort study, Jaeger BR et al. (13) showed combined lipoprotein apheresis and lipid-lowering medication prevent major adverse coronary events more efficaciously than lipid-lowering medication alone. Similarly, the Pro(a) LiFe-study confirmed with a prospective multicenter design that lipoprotein apheresis can effectively reduce Lp(a) plasma levels and prevent cardiovascular events (14). Ezhov MV et al. (15) also showed that isolated extracorporeal Lp(a) elimination over an 18 months period produced regression of carotid intima-media thickness in stable CHD patients with high Lp(a) levels. Although lipoprotein apheresis also decreases other atherogenic lipoproteins, such as oxydized LDL, these results suggest possibilities that lowering $\operatorname{Lp}(\mathrm{a})$ levels might reduce adverse coronary events.

$\mathrm{Lp}$ (a) is not present in experimental animal and $\mathrm{Lp}(\mathrm{a})$ is composed of a moiety with apolipoprotein B-100 (ApoB-100). Expression of apo (a) alone in mice does not form a complex with endogenous mouse LDL, indicating that human LDL is also required to study its function. Therefore, we have to use double transgenic mice with human apo (a) and human LDL to test the Lp(a) function. The previous studies showed that mice expressing the apo(a) gene developed atherosclerosis $(16,17)$. Using this model animal, Kyutoku M et al. (18) reported that DNA vaccine against apo(a) could significantly inhibit neointima formation in carotid artery ligation and concluded that the pro-atherosclerotic actions of $\mathrm{Lp}$ (a) could be prevented by reducing $\mathrm{Lp}$ (a) levels.

More recently, Evolocumab, a monoclonal antibody (mAb) to proprotein convertase subtilisin/kexin type 9 (PCSK9), decreases $\mathrm{Lp}$ (a) (19). The reduction of $\mathrm{Lp}$ (a) levels are thought to be secondary to low circulating LDL due to the increased LDL receptors by this drug. 

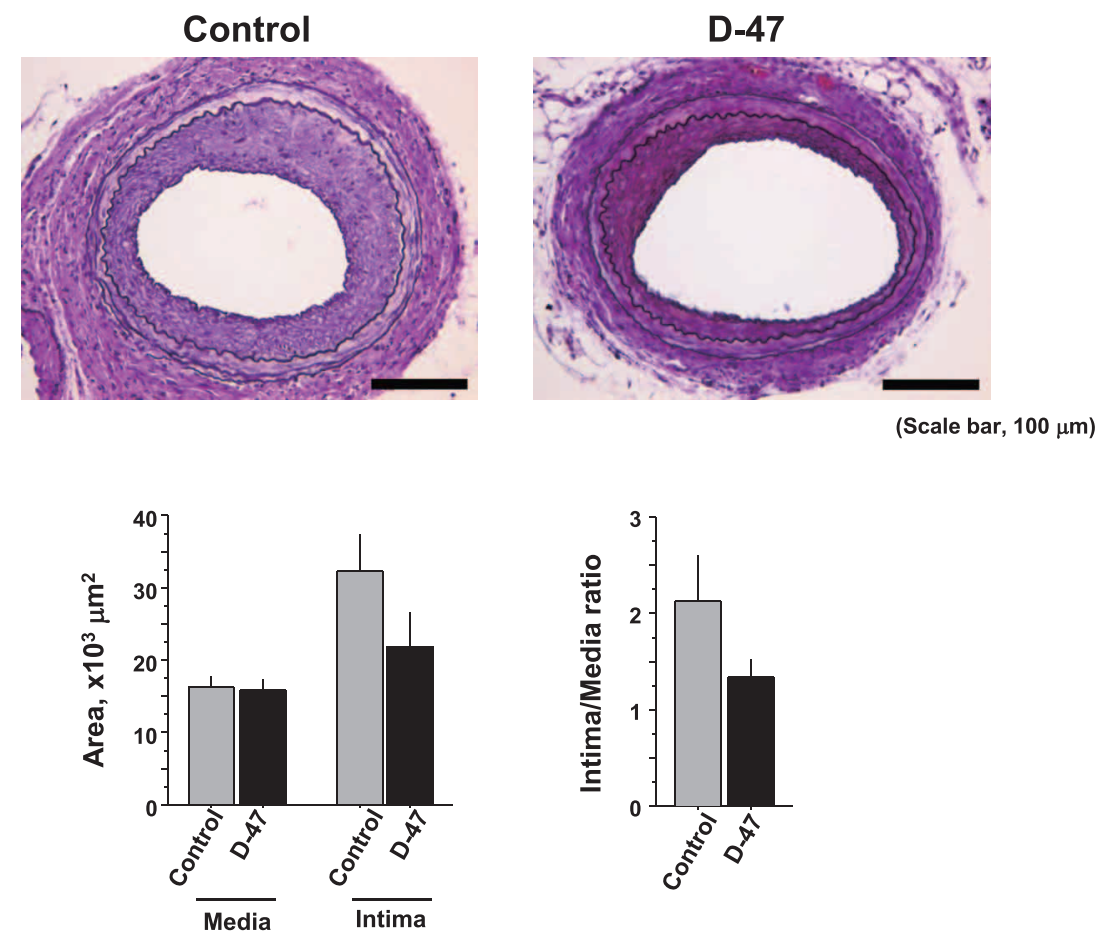

Fig. 2 Effect of D-47 on inhibition of neointima formation.

Upper panels shows femoral arteries stained with Elastica van Gieson. In the mice treated with D-47, the neointima areas, which are defined as the area of the space between the lumen and internal elastic lamina, were smaller than those in control mice. Left lower panel shows the areas of media and intima in each mouse. The area of the media was not different between two groups, but the area of intima was tended to be smaller in D-47 treated mice. Right lower panel shows that the ratio of the neointima area to the media was tended to be smaller in the D- 47 treated mice than in the control mice.

Unfortunately, the high degree of homology between apo(a) and plasminogen has been difficult in the development of drugs against $\mathrm{Lp}(\mathrm{a})$. The drugs which selectively inhibit $\mathrm{Lp}(\mathrm{a})$ is particularly attractive to clinical application. D-47 is a novel drug which selectively lowers serum $\mathrm{Lp}$ (a) levels. Vascular injury model is a useful tools for investigating the mechanism of vascular remodeling because they invoke leukocyte adhesion and infiltration, proliferation of smooth muscle cells and neointima formation $(9,10)$. Using this model and the double transgenic mice, we have shown that D-47 lowered serum levels of $\mathrm{Lp}(\mathrm{a})$ and possibly reduced vascular inflammation and neointima formation after vascular injury. Together with previous studies, the results of the present study suggest lowering of $\mathrm{Lp}$ (a) might reduce cardiovascular diseases related to high $\operatorname{Lp}(\mathrm{a})$.

This is a preliminary study of a new drug and the numbers of the studied animals were small, by which we could not obtain the statistical significance in reduction of neointima formation. Furthermore, the study was tested in model animals which are not identical to human. Therefore, we do not know whether we can extrapolate the results of the study to human with high Lp(a) levels. Further studies will be waited. The results of this study, however, suggest a novel therapeutic strategy for the treatment of cardiovascular diseases that are related to high $\mathrm{Lp}(\mathrm{a})$ levels.

\section{ACKNOWLEDGEMENT}

This study was supported in part by grants-in-aid from Adaptable and Seamless Technology transfer Program through targetdriven R\&D (\# AS242Z02091Q).

\section{DISCLOSURE}

There are no conflicts of interest on D-47 and Lp(a).

\section{REFERENCES}

1. Marcovina SM, Koschinsky ML: A critical evaluation of the role of $\mathrm{Lp}$ (a) in cardiovascular disease : can $\mathrm{Lp}$ (a) be useful in risk assessment? Semin Vasc Med $2: 335-344,2002$

2. Marcovina SM, Koschinsky ML : Evaluation of lipoprotein(a) as a prothrombotic factor: progress from bench to bedside. Curr Opin Lipidol $14: 361-366,2003$

3. Executive summary of the third report of the National Cholesterol Education Program (NCEP) expert panel on detection, evaluation, and treatment of high blood cholesterol in adults (adult treatment panel III). J Am Med 285 : 2486-2497, 2001

4. Lawn RM : How often has Lp(a) evolved? Clin Genet 49 : 167 174, 1996

5. Ohno T, Yano S, Yamada H, Shirasaka T, Yamamoto A, Kobayashi K, Ogawa K : Synthesis of the optical isomers of 4-[1-(4-tert-butylphenyl) -2-oxo- pyrrolidine-4-yl]methyloxybenzoic acid (S-2) and their biological evaluation as antilipidemic agent. Chem Pharm Bull (Tokyo). 47 : 1549-1554, 1999

6. Morishita R, Yamada S, Yamamoto K, Tomita N, Kida I, Sakurabayashi I, Kikuchi A, Kaneda Y, Lawn R, Higaki J, Ogihara T: Novel therapeutic strategy for atherosclerosis : ribozyme oligonucleotides against apolipoprotein (a) selectively inhibit apolipoprotein (a), but not plasminogen, gene expression. Circulation 98 : 1898-1904, 1998

7. Lawn RM, Wade DP, Hammer RE, et al. Atherogenesis in 
transgenic mice expressing human apolipoprotein (a). Nature $360: 670-672,1992$

8. Callow MJ, Verstuyft J, Tangirala R, et al. Atherogenesis in transgenic mice with human apolipoprotein B and lipoprotein (a). J Clin Invest 96 : 1639-1646, 1995

9. Sata M, Maejima Y, Adachi F, Fukino K, Saiura A, Sugiura S, Aoyagi T, Imai Y, Kurihara H, Kimura K, Omata M, Makuuchi M, Hirata Y, Nagai R: A mouse model of vascular injury that induces rapid onset of medial cell apoptosis followed by reproducible neointimal hyperplasia. J Mol Cell Cardiol 32 : 2097-2104, 2000

10. Takaoka M, Nagata D, Kihara S, Shimomura I, Kimura Y, Tabata Y, Saito Y, Nagai R, Sata M : Periadventitial adipose tissue plays a critical role in vascular remodeling. Circ Res 105 : 906-911, 2009

11. Kojima S, Harpel PC, Rifkin DB : Lipoprotein (a) inhibits the generation of transforming growth factor beta : an endogenous inhibitor of smooth muscle cell migration. J Cell Biol $113: 1439-1445,1991$

12. Grainger DJ, Kemp PR, Liu AC, Lawn RM, Metcalfe JC : Activation of transforming growth factor-b is inhibited in transgenic apolipoprotein (a) mice. Nature 370 : 460-462, 1994

13. Jaeger BR, Richter Y, Nagel D, Heigl F, Vogt A, Roeseler E, Parhofer K, Ramlow W, Koch M, Utermann G, Labarrere CA, Seidel D, Group of Clinical Investigators : Longitudinal cohort study on the effectiveness of lipid apheresis treatment to reduce high lipoprotein (a) levels and prevent major adverse coronary events. Nat Clin Pract Cardiovasc Med 6 : 229-239, 2009

14. Klingel R, Heibges A, Fassbender C. : Lipoprotein apheresis results in plaque stabilization and prevention of cardiovascular events : comments on the prospective Pro(a)LiFe study. Clin Res Cardiol 10(Suppl.) : 46-50, 2015

15. Ezhov MV, Safarova MS, Afanasieva OI, Pogorelova OA, Tripoten MI, Adamova IY, Konovalov GA, Balakhonova TV, Pokrovsky SN : Specific Lipoprotein(a) apheresis attenuates progression of carotid intima-media thickness in coronary heart disease patients with high lipoprotein(a) levels. Atheroscler 18(Suppl) : 163-9. 2015

16. Maeda S, Abe A, SeishimaBrown G, Albers JJ, Fisher LD, Schaefer SM, Lin JT, Kaplan C, Zhao XQ, Bisson BD, Fitzpatrick VF, Dodge HT : Regression of coronary artery disease as a result of intensive lipid lowering therapy in men with high levels of apolipoprotein B. N Engl J M 323 : 12891298, 1990

17. Callow MJ, Stoltzfus LJ, Lawn RM, Rubin EM : Expression of human apolipoprotein B and assembly of lipoprotein (a) in transgenic mice. Proc Natl Acad Sci U S A. 91 : 2130-2134, 1994

18. Kyutoku M, Nakagami H, Koriyama H, Nakagami F, Shimamura M, Kurinami H, Tomioka H, Miyake T, Katsuya T, Morishita R: Inhibition of neointima formation through DNA vaccination for apolipoprotein (a) : a new therapeutic strategy for lipoprotein(a). Sci Rep 3 : 1600, 2013 doi : 10.1038/ srep01600

19. Raal FJ, Giugliano RP, Sabatine MS, Koren MJ, Blom D, Seidah NG, Honarpour N, Lira A, Xue A, Chiruvolu P, Jackson S, Di M, Peach M, Somaratne R, Wasserman SM, Scott R, Stein EA : PCSK9 inhibition-mediated reduction in Lp(a) with evolocumab : an analysis of 10 clinical trials and the LDL receptor's role. J Lipid Res 57 : 1086-1096, 2016 